\title{
ARCHITECTURAL MODELING WITH REUSABLE INTERACTIVE 3D OBJECTS
}

\author{
Jacek Chmielewski \\ Department of Information Technology \\ Poznan University of Economics \\ Poznan, Poland \\ E-mail: Jacek.Chmielewski@kti.ae.poznan.pl
}

\section{KEYWORDS}

Virtual Reality, modeling, interactive 3D objects, interaction model, metadata, XML.

\begin{abstract}
$3 \mathrm{D}$ modeling is extensively used in many engineering fields, notably in architecture. 3D models of new buildings are built with 3D objects taken from object libraries provided by producers of real objects (i.e. windows, lifts, etc). Such libraries can also include interactive 3D objects that are not only static geometrical representations, but also behave like their real counterparts. Searching for such interactive object in a large library requires object metadata describing object interaction properties. Existing metadata standards are not sufficient for describing object interactions. In this paper a new solution for interactions metadata is presented. The new solution is composed of concepts and tools for modeling an interaction, describing different aspects of an interaction, building XML documents with interaction metadata and using information contained in these documents to search specific objects. The usage of all those concepts and tools is presented in a detailed scenario from the field of architectural modeling.
\end{abstract}

\section{INTRODUCTION}

Modeling techniques based on virtual reality and 3D representations of real objects are widely used in mechanical design and architecture, among others. The time required to build such 3D models can be greatly decreased by the use of libraries of predefined components, which can be simply reused instead of designing them from scratch for every new model. This fact is especially visible in architectural modeling, where different elements of a newly designed building (i.e. windows, doors, lifts, etc) are usually provided by producers of these elements.

3D models of new buildings are often used not only for designing purposes, but also for generating visualizations and publications for marketing purposes. Often, such marketing visualizations include fly-by animations or presentations of specific features. However, it is possible to go a step further and provide potential clients and end-users with 3D immersive and interactive experience, by allowing them to walk through their new office, shop or apartment before it actually gets built. Such approach can be used not only for demonstration, but also for testing the newly designed space and optimizing some features, like lighting conditions, ergonomics, etc.

$3 \mathrm{D}$ interactive model requires components (i.e. reusable objects) that are not only geometrical representations of their real counterparts, but are also interactive and behave like real objects. Such interactive objects (Walczak 2006) can be also provided by producers of real elements in a form of object libraries, in the same way as they do it with static objects. However, searching for interactive objects in large object libraries is more complex. With static 3D objects it is enough to have some classification of objects and simple search engine that is capable of using this classification as object semantic description, along with geometry characteristics extracted directly from object 3D geometry. With interactive 3D object there is one additional element that has to be taken into account: object behavior. For interactive presentations, object behavior is usually the most important element, due to the fact that object interaction characteristic is used by designer to decide whether the object is suitable for the new design. In most cases object interaction characteristics cannot be inferred from the object definition. It is required to introduce additional description about the object - metadata. Metadata capable of describing all possible interactions of an object and will allow formulate queries that address specific interaction properties.

\section{BACKGROUND}

The topic of multimedia object metadata is well researched and developed. There are a number of metadata standards that can be used to describe object general properties or object format and purpose specific characteristics. The most general metadata standard is Dublin Core, which is a set of fifteen elements designed to foster consensus across disciplines for the discoveryoriented description of diverse resources in an electronic environment. Dublin Core Metadata Element Set (DCMES 2008) is intended to support cross-discipline resource discovery and it does not satisfy every possible declarative need in every discipline. Thus, in most applications it is used in a combination with more advanced, domain-specific metadata standard. DC elements are usually a subset of resource metadata and 
are used as a minimal metadata for data exchange and discovery.

Interactive 3D objects usually contain many multimedia components like: images and video as textures, audio, text, complex 3D geometry, therefore the complete description of such objects may be composed of various domain specific metadata. For still images EXIF (EXIF 2002), DIG35 (DIG35 2000) or NISO Z39.87 (Z39.87 2001) can be used. Video can be described with AAF (AAF 2005), MXF DMS-1 (MXF 2004) or P/Meta (P/Meta 2007). Audio components of the interactive 3D object can use MusicBrainz (Swartz 2001) or simple ID3 tags (ID3 2000). However, complex multimedia resources like interactive $3 \mathrm{D}$ objects are usually described with the most universal metadata standard for multimedia objects - MPEG-7 (Martinez 2004; ISO/IEC 15938). It addresses not only general and format specific information, but also object semantics, geometry and spatio-temporal composition. MPEG-7 was formulated with audio and video contents in mind and its handling of 3D objects is limited. Nevertheless, in the past few years, some 3D metadata solutions were developed (Lorenz at al. 2006; Pitarello and de Faveri 2006; Bilasco et al. 2005). Especially worth mentioning, is the 3D SEmantics Annotation Model [3DSEAM] (Bilasco et al. 2006) developed by a group from Laboratoire Informatique de Grenoble [LIG], lead by Ioan Marius Bilasco. 3DSEAM extends MPEG-7 localization descriptors with Structural Locator and 3D Region Locator tools, making MPEG-7 the best available metadata solution for 3D multimedia objects.

However, even with such great pool of metadata standards and solutions like MPEG-7 there is no universal and extensible solution capable of describing interaction properties of interactive $3 \mathrm{D}$ objects. It is due to the fact that interactive objects change their state according to interaction results and interaction context, and new object parameter values cannot be determined a priori. Existing metadata solutions where designed to handle non-interactive resources and are focused on a single state of the described object. Therefore they cannot be efficiently used for interaction metadata

\section{INTERACTION METADATA}

As stated in the previous section, existing metadata solutions like for example MPEG-7 with 3DSEAM extension (Bilasco et al. 2006), can be used for describing object semantics and geometry. However they are not sufficient for describing object interactions. Existing metadata standards for multimedia objects were designed to describe a static or linear content, i.e. still images, documents or movies. In case of interaction descriptions the problem is more sophisticated. An interaction is not a simple 'content'. Interaction influences modification of the content, i.e. the object, but interaction itself is an action based on object behavior. The behavior is represented as an algorithm expressed by a computer program. The interaction metadata does not have to describe the whole algorithm, which may be very complex or confidential. To enable analyzing object interaction properties and chains of subsequent interactions it is enough to describe only the result of execution of such computer program. Existing metadata solutions are not applicable for describing multiple states of an object (i.e. all possible execution results). Therefore there is a need for a new metadata solution.

To deal with this situation, a new approach to interaction metadata is proposed. The proposed approach is based on following assumptions. To be interactive, objects need to have a behavior. Behavior is encoded in a form of a computer program. Computer program of an interactive object has at least one communication interface - an API, which allows exchanging information with the $3 \mathrm{D}$ virtual world or other interactive objects. Such API includes a number of functions and attributes that reflect the state of an object. Knowing the API and the computer program it is possible to precisely describe object interaction characteristics and possible object interactions, as well as match compatible objects. Following assumptions stated above, two new concepts are introduced: Interaction Interfaces and Multimedia Interaction Model. These concepts are accompanied with one additional tool called Interaction Metadata Query subLanguage.

\section{Interaction Interface}

The Interaction Interface concept is used to describe the interface of an object in terms of functions and attributes related to object interactions. Both elements of the interface are represented by object parameter types of the Interactive Interface and are accompanied with semantic descriptions. The Interaction Interfaces are used in metadata as object interaction characteristic and as a dictionary of parameter types referenced in the Multimedia Interaction Model.

An interaction interface groups object parameter types that have similar semantics, e.g. object parameter types related to object visual characteristics or object parameter types related to object internal components. An object implements a given II if the set of object parameter types is a superset of the set of parameter types of this II.

The proposed solution provides Interaction Interface Definition Rules that describe how to define new object parameter types and how to group them in new interaction interfaces. Single object parameter type definition is composed of an identifier, specification of a data type of values of object parameters of this object parameter type and optional specification of arguments required to evaluate the object parameter function. The technical part of parameter type definition is accompanied with semantic description or relation to an external ontology. While the technical part is required to build metadata, the semantic part is needed to allow search engines properly interpret interaction metadata and match object technical parameters with a semantic query. 


\section{Multimedia Interaction Model}

The Multimedia Interaction Model is a conceptual model of an interaction described from the point of view of an object. It means that the interaction description is build around object reaction and a context that triggers the reaction. This approach comes from areas of computer science dealing with active environments, especially active databases. Research on the active databases resulted in paradigm called Event-ConditionAction (ECA) (Dayal U. et al 1988), which was introduced in late eighties by the members of the HiPAC project. The semantics of ECA rules are straightforward: when an event occurs, evaluate the condition associated with the event; and, if the condition is satisfied, trigger the action. The ECA rules are used up to date to express active DMBS features and eventreaction oriented features in other systems (Bry and Patrânjan 2005; Papamarkos et al. 2003; Thome et al. 2005; Zhou et al. 2004). The way the ECA paradigm is used in the Multimedia Interaction Model is the following. The Event element corresponds to the trigger of an interaction. The trigger is a change of a selected property of some object or a virtual environment in which the interaction occurs. The Condition element describes the context of an interaction. The context represents a state of selected objects or the virtual environment that is required to allow the action. The Action element matches the object reaction. The reaction describes how the object changes itself in a reply to the event in a given context.

\section{Event}

An event is a change of the state of an object. The object is called: trigger object. In the MIM model, the Event component contains a set of preconditions that need to be satisfied to trigger a particular interaction. The preconditions include a specification of a 3D interaction space and a non-empty set of object parameter types. To satisfy preconditions, the event has to occur inside a specified 3D interaction space and object parameters, which values are modified, have to belong to one of specified object parameter types.

\section{Condition}

A condition is a set of requirements set on the states of trigger object, the environment and interacting object. A condition needs to be satisfied to trigger an action. In the Condition component of the MIM model, a mathematical description has a form of logical expression. The logical expression represents all condition requirements. Semantic description of a condition may be used as a substitute or an enhancement of the mathematical description. The Condition element of MIM metadata is optional and can be omitted. In such case an interaction will be executed at each occurrence of the event.

\section{Action}

An action is a change of the state of an interacting object. In the MIM model, the Action component describes the outcome of an action, a new object state, expressed as a semantic description optionally accompanied by mathematical description represented by mathematical expressions used to calculate new values of object parameters. An action can be composed of an arbitrary number of sub-actions that provide more detailed descriptions. Such approach allows building action descriptions that can be evaluated on different levels of details.

\section{Interaction Metadata Query sub-Language}

The Interaction Metadata Query sub-Language goal is to provide easy way to access and use information available in object interaction metadata. IMQL provides a set of operators that can be used in simple keyword comparisons and advanced semantic searches. IMQL syntax relates directly to the MIM model structure and provides references to interaction interfaces of compared objects and parameter types of these objects. Object matching queries can be complex and difficult, especially in the field of interactions. The use of interaction interfaces facilitates object matching queries. The IMQL integrates with interaction interface definitions enabling the use of new object parameter types and value data included directly in the query. IMQL is composed of two parts: first, defining the source of object metadata; second, specifying filtering criteria. The first part can be replaced by results of another search query, while the output of the filtering criteria can be passed for further processing. Such solution allows embedding an IMQL query within larger query formulated in a different query language and performing a complex search by a single search system.

The MIM metadata has the most expression power when combined with more general multimedia metadata standards, like for example MPEG-7. Therefore the IMQL has to be used in conjunction with query languages capable of processing these general metadata standards. As mentioned, the IMQL can be embedded in queries written in other query languages forming a complex query. Complex queries enable search systems to look into standard metadata descriptions and interaction descriptions in a single process.

To provide universality of IMQL embedding rules, an IMQL query is embedded as a function. The imql() function takes object metadata URI and IMQL WHERE clause contents as arguments. The function returns Boolean value equal TRUE if given metadata matches the specified IMQL where_expr. IMQL function syntax is the following:

imql (object_metadata_URI, where_expr)

Embedding IMQL query as a function allows including IMQL in an arbitrary query language: from XML 
related XQuery, to RDF query language SPARQL, to $\mathrm{SQL}$ used for querying relational databases.

\section{SEARCHING FOR INTERACTIVE OBJECTS}

Consider an architecture studio where engineers work to design new buildings. Each building design is composed of many components, like for example walls, windows, doors, lifts, stairs, etc. All these components are virtual models of real objects that will be used during the development of a new building. Some of these components can be interactive and have embedded behavior that makes this object act like its real counterpart. Example of an architectural object with embedded behavior is a model of automatic 'sliding door'. Consider a 'sliding door' model that opens itself when someone approaches the door and closes itself automatically. It can be locked in open or closed state, which means that the door can be permanently closed or permanently open. The actions of door opening and closing are visualized as animations and complemented with appropriate sound. The diagram of such object is presented in Figure 1.
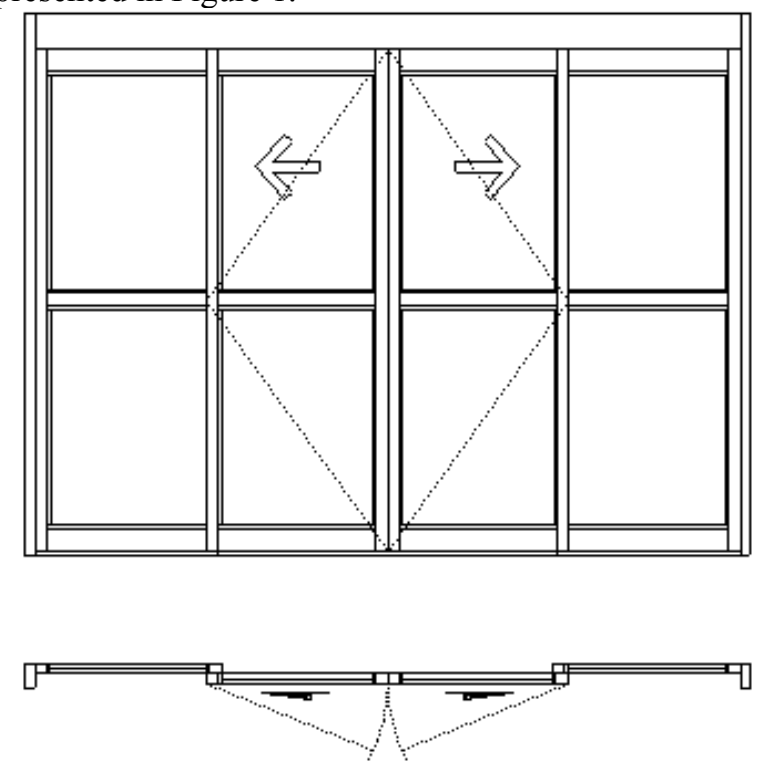

Figure 1: 'Sliding door' diagram

The 'sliding door' virtual model has two interactions: 'open' and 'close'. First interaction is triggered by an object moving in the area monitored by door motion sensor. Second interaction, 'close', is triggered by an object leaving the monitored area. The trigger object of both interactions can be any object big enough to exceed the sensitivity limit of the door motion sensor. The 'sliding door' can also be locked in the closed or open state. The locked parameter is a representation of object internal state and there is no specific interaction for changing the value of the locked parameter.

Parameters used in the 'open' and 'close' interactions include PositionCenter, SizeBoudingBox and PlayedSound from general 'Visual Interaction Interface' [V-II] and 'Aural Interaction Interface' [A-II], and Closed and Locked parameters from a custom interaction interface called 'ArchItems - Door
Interaction Interface' [AIdoor-II]. The AIdoor-II interaction interface defines the Closed and Locked parameters as binary flags with Boolean data type. Both, A-II and AIdoor-II interfaces are fictitious and introduced specially for this scenario. V-II is a general predefined interaction interface.

Both interactions of the 'sliding door' object are similar. This example describes in detail the first interaction 'open'. The Event metadata element of the 'open' interaction contains specification of a parameter whose value change triggers the event, and definition of the $3 \mathrm{D}$ interaction space. The event of the 'open' interaction is triggered by a change of the PositionCenter parameter of an object located inside the 3D interaction space. The $3 \mathrm{D}$ interaction space has a shape of a cone with the apex located above the door and the vertical axis directed down at an angle of $45^{\circ}$. Excerpt of XML document with Event element description is presented in Listing 1.

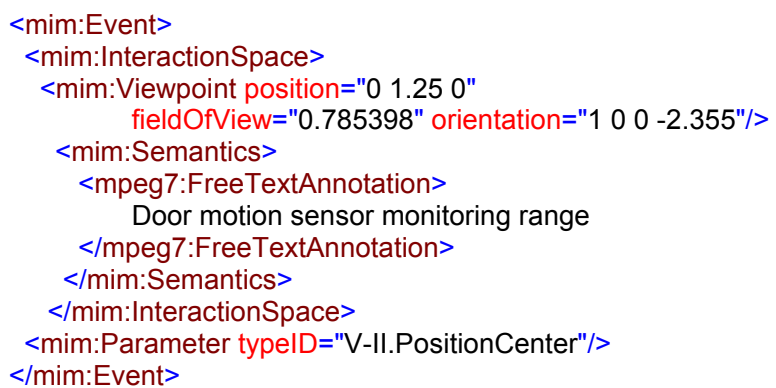

Listing 1: Excerpt of XML metadata of the 'open' interaction: Event element

The Condition element of 'open' interaction metadata contains a mathematical expression which checks if the size of the trigger object is above the threshold required to execute the interaction. The size parameter value is bound to the $\mathrm{S}$ symbol, while the threshold is represented by a numeric value 0.32 . The mathematical expression written MathML refers to single components of object size (S) and compares its product with the threshold value.

$$
\text { S.X } \cdot \text { S.y } \cdot \text { S.z }>=0.32
$$

Other conditions that have to be met to execute the 'open' interaction refer to the state of the 'sliding door'. The door has to be closed and not locked. These conditions refer to Closed parameter value bound to $\mathrm{C}$ symbol and Locked parameter value bound to $\mathrm{L}$ symbol. XML representation of the Condition metadata element is the following.

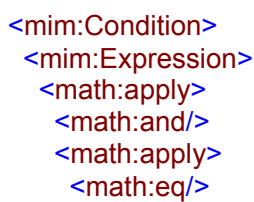




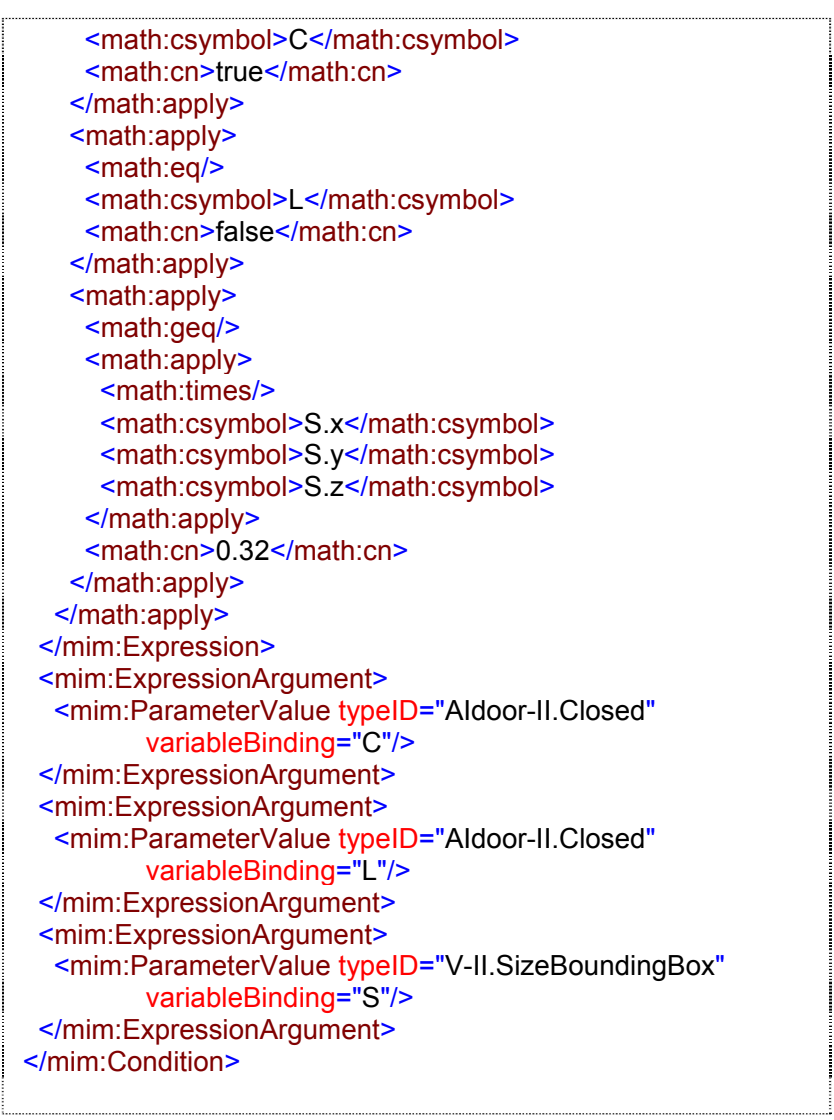

Listing 2: Excerpt of XML metadata of the 'open' interaction: Condition element

As mentioned in the previous section, the description of the MIM Action element can be composed of an arbitrary number of sub-actions. The interaction metadata of 'open' interaction uses such complex structure. It consists of descriptions of two parallel actions. First action is an animation of door opening. Second action is a sound that occurs when the door is opening. The animation action is described by a simple semantic description: "Door opens slowly to the sides". The action related with sound includes sound characteristics described by value of parameter taken from a custom Aural Interaction Interface. The value of this parameter is represented in the MPEG-7 AudioSpectrumBasisType. Listing below illustrates the XML representation of the Action element.

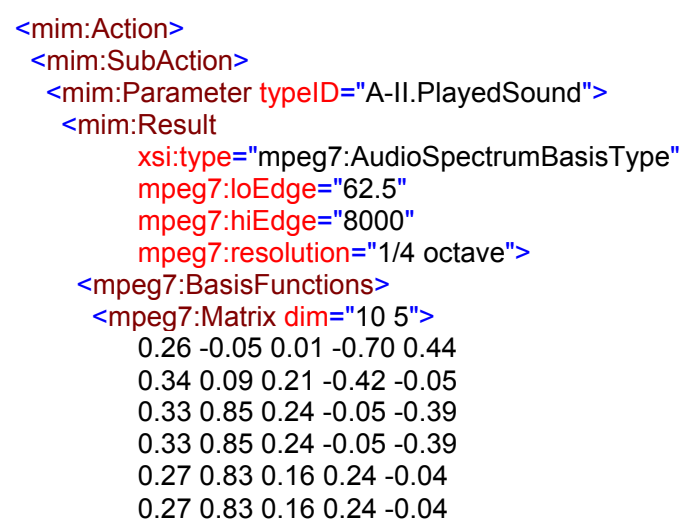

0.230 .830 .090 .270 .24
0.200 .830 .040 .220 .40
0.170 .810 .010 .140 .37
0.33 -0.15 0.24 0.050 .39
</mpeg7:Matrix>
</mpeg7:BasisFunctions>
</mim:Result>
</mim:Parameter>
</mim:SubAction>
<mim:SubAction>
<mim:Semantics>
<mpeg7:FreeTextAnnotation>
Door opens slowly to the sides.
$</$ mpeg7:FreeTextAnnotation>
</mim:Semantics>
$</$ mim:SubAction>
$</$ mim:Action>

Listing 3: Excerpt of XML metadata of the 'open' interaction: Action element

Consider a repository of interactive 3D models of door manufactured by a certain company. Each object consists of a 3D geometry, embedded behavior, and MPEG-7 metadata with embedded MIM metadata. Such repository is accessible to architects designing new buildings. Architect specifies door properties that are required by his/her design and looks for doors that match these properties. A simple query could look for interactive 3D objects tagged with 'door' and 'automatic' keywords and reacts to a change of position of other objects. Such query can be formulated in the following way.

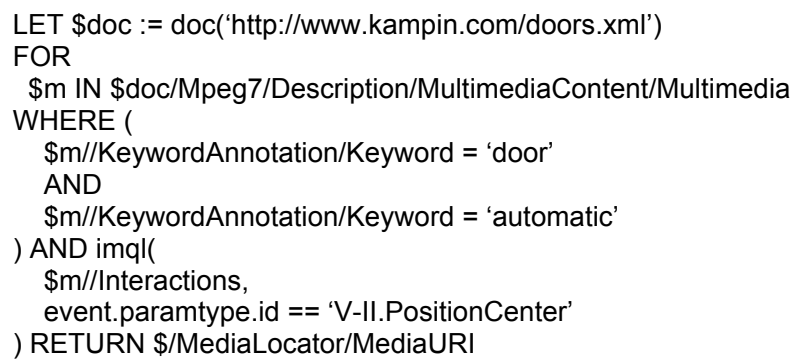

Listing 4: Sample XQuery query with embedded IMQL query

The architect can also specify requirements on the area of the motion sensor. For example a set of points may be specified that have to be included in the sensor area. Points have to be specified in the X3D SFVector3f format (X3D). For example: furthest point on the ground ( $\left.\begin{array}{lll}0 & 0 & -5\end{array}\right)$ and at half of the door height $\left(\begin{array}{lll}0 & 1 & -3\end{array}\right)$, and furthest points located to the sides of the door (-1 1 $-1)$ and (1 $1-1)$. The previous query extended with such requirement is the following.

LET \$doc := doc('http://www.kampin.com/doors.xml')

FOR

\$m IN \$doc/Mpeg7/Description/MultimediaContent/Multimedia WHERE (

$\$ \mathrm{~m} / /$ KeywordAnnotation/Keyword = 'door' 


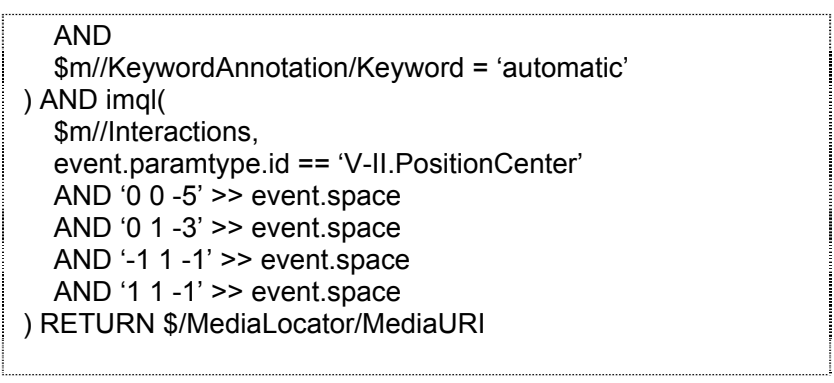

Listing 5: Query presented in Listing 4 extended with $3 \mathrm{D}$ interaction space requirement

Moreover, also detailed action properties can be included in the query. Assuming that an architect wants to find all sliding doors with a specific opening sound, the query is the following.

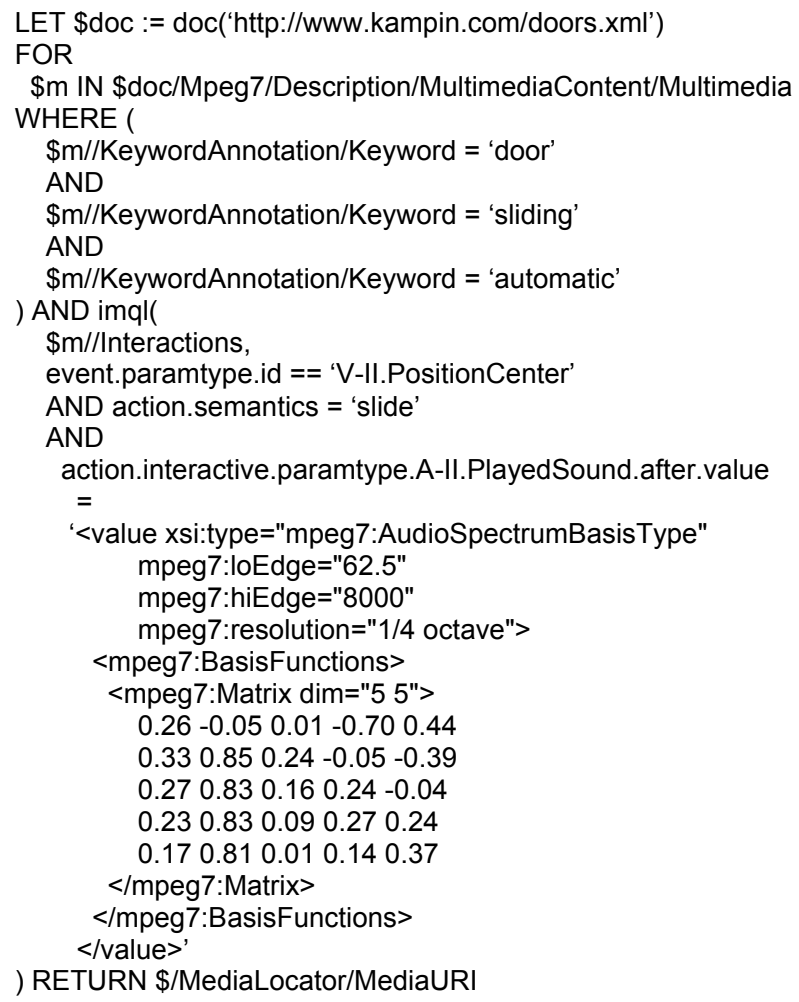

Listing 6: Query presented in Listing 4 extended with sub-action requirements

\section{CONCLUSIONS}

The three elements presented in the paper form a complete solution for describing object interaction characteristics and exploiting such descriptions. Although, the approach is particularly useful with architectural modeling, is not specific to any particular domain. With the use of Interaction Interface concept it can be easily employed for describing 3D representations of body parts used in medicine or describing some interactive objects in game development industry. With small modifications, namely definition of new interaction interfaces, the presented solution for building interaction metadata can be even more general. It can be used to describe any interactive entity, even if it is not a $3 \mathrm{D}$ object, for example a role in automatic negotiations or a software component.

The domain of interaction metadata for autonomous, reusable interactive $3 \mathrm{D}$ objects is in early stages of development. There are many areas open for research. First and most obvious area is research of search engines and comparison algorithms for interaction properties. Second is related with interaction properties descriptors. Some of such descriptors could be calculated directly from the object definition making the metadata creation process faster and more efficient. Moreover, different applications will require different interaction interfaces. Therefore the third area of potential research is related with development of universal interaction interfaces and tools for publishing and discovery of such interfaces. With such tools producers of sliding doors could post libraries of 3D representations of their products that are described with a domain specific interaction interfaces. Making these libraries searchable and allowing for CAD system extensions that connect with object libraries spread through the Internet and automatically find objects best suited for a given design, which could make designer life even easier.

\section{REFERENCES}

AAF, AAF Object Specification v1.1, 2005, Available: http://www.aafassociation.org/html/techinfo/index.html

Bilasco I.M., Gensel J., Villanova-Oliver M., Martin H., On indexing of 3D scenes using MPEG-7, in Proceedings of ACM Multimedia 2005, Singapore, 2005, pp: 471-474

Bilasco I.M., Gensel J., Villanova-Oliver M., Martin H., An MPEG-7 framework enhancing the reuse of 3D models, in Proceedings of Web3D Symposium 2006, Columbia, Maryland USA, 2006, pp: 65-74

Bry F. Patrânjan P., Reactivity on the web: paradigms and applications of the language XChange, in Proceedings of the 2005 ACM Symposium on Applied Computing, Santa Fe, New Mexico USA, 2005, pp: 1645-1649

Dayal U. et al., HiPAC: a Research Project in Active, TimeConstrained Database Management, Interim Report, Technical Report XAIT-88-02, Xerox Advanced Information Technology, June 1988

DCMES: Dublin Core Metadata Element Set, Version 1.1, 2008, Available: http://dublincore.org/documents/dces/

DIG35: Digital Imaging Group, DIG35 Specification, Metadata for Digital Images 1.0, 2000, Available: $\mathrm{http}: / / \mathrm{xml}$.coverpages.org/FU-Berlin-DIG35-v10Sept00.pdf

EXIF: Japan Electronics and Information Technology Industries Association (JEITA) Standard, JEITA CP-3451, Exchangeable image file format for digital still cameras: Exif Version 2.2, 2002, Available: http://www.exif.org/Exif2-2.PDF

ID3, ID3 Informal standard, ID3 tag version 2.4.0, 2000, Available: http://www.id3.org/id3v2.4.0-frames

ISO/IEC 15938, ISO International Standard, Information technology - Multimedia content description interface Available: http://www.iso.org/iso/en/prods-services/ popstds/mpeg.html 
Lorenz B., Ohlbach H.J., Stoffel E.P., A Hybrid Spatial Model for Representing Indoor Environments, in Proceedings of W2GIS 2006, (LNCS 4295), Hong Kong, China, 2006, pp: 102-112

Martinez J. M. et al., MPEG-7 Overview, 2004, Available: http://www.chiariglione.org/MPEG/standards/mpeg7/mpeg-7.htm

MXF: SMPTE 377M-2004 Television Material Exchange Format (MXF) Standard - File Format Specification, 2004, Available: http://store.smpte.org/category-s/1.htm

Papamarkos G., Poulovassilis A., Wood PT., Event-ConditionAction Rule Languages for the Semantic Web, Workshop on Semantic Web and Databases, 2003

Pitarello F., de Faveri A., Semantic Description of 3D Environments: a Proposal Based on Web Standards, in Proceedings of Web3D Symposium 2006, Columbia, Maryland USA, 2006, pp: 85-95

P/Meta: EBU Metadata Exchange Scheme (EBU P/Meta) 2.0, EBU Tech 3295-v2, 2007, Available: http://www.ebu.ch/en/technical/metadata/specifications/in dex.php

Swartz A., MusicBrainz: A Semantic Web Service, 2001 Available: http://logicerror.com/musicbrainzArticle

Thome B., Gawlick D., Pratt M., Event processing with an oracle database, in Proceedings of the 2005 ACM SIGMOD International Conference on Management of Data, Baltimore, Maryland USA, 2005, pp: 863-867

Walczak K., Beh-VR: Modeling Behavior of Dynamic Virtual Reality Contents, in Proceedings of the 12th International Conference on Virtual Systems and Multimedia VSMM 2006, in: H. Zha et al. (Eds.): Interactive Technologies and Sociotechnical Systems, Lecture Notes in Computer Sciences 4270, Springer Verlag Heidelberg 2006, pp. 4051

X3D Specifications

Available: http://www.web3d.org/x3d/specifications/

Z39.87: NISO American National Standard, ANSI/NISO Z39.87-2006, Data Dictionary - Technical Metadata for Digital Still Images, 2001, Available: http://www.niso.org/kst/reports/standards/

Zhou X., Zhang S.,Cao J., Dai K., Event Condition Action Rule Based Intelligent Agent Architecture, Journal of Shanghai Jiaotong University, Volume 38, Issue 1, January 2004, pp: 14-17

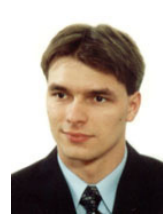

JACEK CHMIELEWSKI was born in Poznan, Poland and went to the Poznan University of Technology, where he studied teleinformatics and obtained his degree in 2001. After the studies he joined the Department of Information Technology at the Poznan University of Economics. In 2008 he received $\mathrm{PhD}$ in the field of multimedia systems. His research interests include multimedia semantics and mobile multimedia. His e-mail address is: Jacek.Chmielewskieae.poznan.pl and his Webpage can be found at http://www.kti.ae.poznan.pl/jacek_chmiele wski.html 